\title{
EFFECT OF REACTION RATE PERIODICITY ON DETONATION PROPAGATION
}

\author{
Eric O. Morano and Joseph E. Shepherd
}

\author{
Graduate Aeronautical Laboratories, California Institute of Technology, Pasadena, CA 91125
}

\begin{abstract}
As an alternative to homogeneous reaction rates, we implement "synthetic" hotspots through a depletion rate that is a function of the local pressure multiplied by a periodic function of the spatial coordinates. We investigate through numerical simulations how the detonation propagation is affected by the heterogeneous rate.
\end{abstract}

\section{INTRODUCTION}

Numerical simulation of shock and detonation physics in explosives can be carried out at various levels of sophistication but must include the essential features of unsteady compressible flow with shock waves, equations of state for reactants and products, and some model of the chemical reaction processes.

However, modeling of high explosives is not straightforward. There are a number of unresolved issues in modeling solid explosives and as a consequence, there is no universally accepted model for the equations of state and the reaction process. One key issue is that solid high explosives often come in the form of pressed powders and for continuum models, this microstructure has always been modeled through an empirical reaction rate. There is no general agreement on how to construct such a rate. Other key issues include the thermodynamic models of the reactants and products. Various approximate models exist but there is no accepted and accurate representation of all thermodynamic states significant to the detonation process. Finally, the chemical reactions occur in a dense fluid environment and it is clear that the reaction models used for ideal gases are deficient in not accounting for the effects of neighboring molecules on the reaction processes.

In this paper, we use a simple model for the equation of state as introduced in [1] and we study the issue of heterogeneity in reaction rate.

\section{ENGINEERING MODELS AND ISSUES}

Many solid explosives are not homogeneous like gases or liquids; the explosive is a granulated material which is coated with a binder, an elastomer, and pressed.

Due to the granular structure, the reaction process is expected to be dominated by the presence of hot-spots, regions of intense reaction created at voids, grain boundaries, and defects. Such regions of intense reaction are the result of locally high temperature due to void collapse, friction of HE crystal surfaces, shock focusing, etc [2].

The classical approach to modeling these explosives is to completely homogenize the material and represent it as a simple fluid that is an equilibrium mixture of reactants and products. Two models of this type are Lee and Tarver [3] and Johnson et al. [4]. The advantage of this approach is that the standard single-fluid hydrodynamic (Euler) equations govern the flow evolution. This approach enables the use of tradi- 
tional hydrocodes as well as more modern approaches based on Riemann solvers such as the Roe-Glaister [5] scheme used in our study.

We follow this approach in our study but avoid the complexity of a mixture equation of state by using a model based on the MieGrüneisen form extended to include heat release.

$$
P(e, v) \sim \mathcal{G} \frac{\left(e-e_{\mathcal{H}}(v)\right)}{v}+P_{\mathcal{H}}(v)+\mathcal{G} \frac{\lambda q}{v},
$$

where internal energy $e_{\mathcal{H}}$ and pressure $P_{\mathcal{H}}$ are obtained from the shock jump conditions using a linear $U_{s}-u_{p}$ relationship, $U_{s}=c_{0}+s u_{p}$. For expanded states, we use a treatment very similar to the pseudo-elastic approximation of Miller and Puckett [7]. We have chosen the following parameters to imitate an "HMX-like" explosive: $\rho_{0}=1.891 \mathrm{~g} / \mathrm{cc}, c_{0}=3.07 \mathrm{~km} / \mathrm{s}, s=1.79$, $\mathcal{G}=0.70$, and $q=14.70 \mathrm{~kJ} / \mathrm{g}$. This results in a CJ velocity of $9.59 \mathrm{~km} / \mathrm{s}$ and a von Neumann pressure $P_{V N} \sim 66.17 \mathrm{GPa}$.

The issue we are studying is the role of explicit reaction rate spatial dependence. Existing models $[3,4]$ homogenize the rate and treat the presence of hot-spots by terms in the reaction rate that represent the initiation or creation of hot-spots and the subsequent growth. In the present study, we have introduced an explicit spatial dependence of the reaction rate and have carried out numerical experiments to determine the effect on detonation initiation and propagation simulations. Since this development is motivated by the role of hot-spots in HE, we refer to these as "synthetic hot-spots". We use a pressure-dependent, single-step depletion law with a fractional order,

$$
R(\lambda, P)=K(\mathbf{x})\left(\frac{P}{P_{V N}}\right)^{\nu}(1-\lambda)^{1 / 2},
$$

where $\nu=5$. The synthetic hot-spot model consists of making $K$ a function of the spatial coordinate $\mathbf{x}$, i.e. a property of the material. Consequently, $K$ must be advected with the particle velocity. The reactive Euler equations are augmented by the following equation:

$$
\frac{\partial}{\partial t}(K \rho)+\nabla \cdot(K \rho \mathbf{u})=0
$$

and solved with a Roe-Glaister solver with passive scalar advection capability.

\section{ONE-DIMENSIONAL NUMERICAL SIMULATIONS}

The steady ZND solution is computed first with a homogeneous rate $K(\mathbf{x})=0.8 \mu \mathrm{s}^{-1}$; its reaction zone length is $\Delta Z_{T}$. The Eulerian computational domain is a $25 \Delta Z_{T}$ long channel with grid-spacing that gave 100 cells in the reaction zone. The profile is used to initialize the Eulerian solver which is run until convergence. The converged profile has a reaction zone length $\Delta Z_{C} \sim \Delta Z_{T}$ and a detonation velocity $U \sim 9.48 \mathrm{~km} / \mathrm{s}$. The slight deficit is due to the small amount of reaction that occurs within the numerical shock front. Experiments with different numbers of cells in the reaction zone indicate that $U \rightarrow U_{C J}$ as $K \rightarrow 0$ for a fixed number of cells in the reaction zone. This converged profile serves as initialization for all the following experiments. Our model spatial dependence

$$
K(\mathbf{x})=0.8(1.0+\sigma|\cos (\alpha x) \cos (\beta y)|)
$$

for this first study is a simple periodic function.

In this paper, we will present only results from one-dimensional simulations, i.e. $\beta=$ 0 . $K(\mathbf{x})$ varies between a minimum value of $0.8 \mu \mathrm{s}^{-1}$ and a maximum value of $(1.0+\sigma) \mu \mathrm{s}^{-1}$. We investigate the effect of each parameter $\sigma$ and $\alpha$ separately.

The first set of numerical experiments we carried out examined the issue of how the spatial dependence of the reaction rate affects the propagation of a near-CJ wave. For a steady wave with a homogeneous rate, the propagation velocity is only a function of the energy released and the rear boundary condition. Since the spatial dependence of the rate will introduce unsteadiness in the flow, it is not clear how this will influence the propagation speed.

The results of a typical simulation are shown in Fig. 1 through 3. The spatial dependence of the reaction rate creates a wave train that propagates backward from the front (Fig. 1 and more clearly in Fig. 3). The interaction of forward 
propagating waves within the reaction zone results in oscillations in the leading shock strength, visible in Fig. 3.

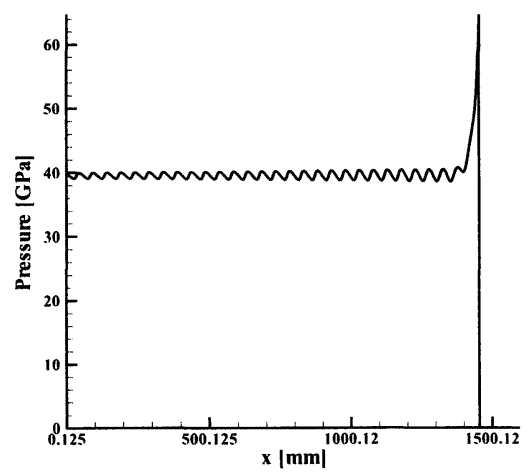

FIGURE 1. Pressure profile @ $t \sim 144.36 \mu \mathrm{s}(\sigma=1.0$ and $\Pi=2.0)$.

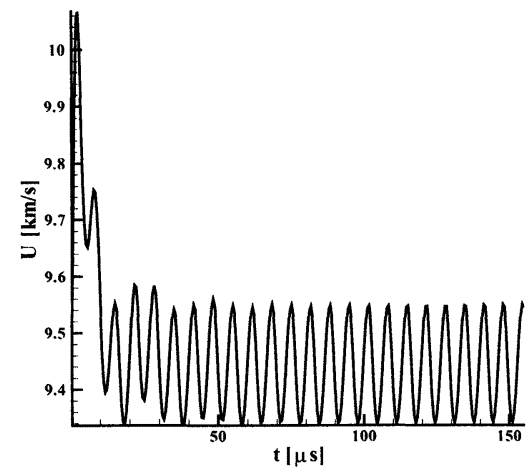

FIGURE 2. Front velocity as a function of time ( $\sigma=$ 1.0 and $\Pi=2.0$ ).

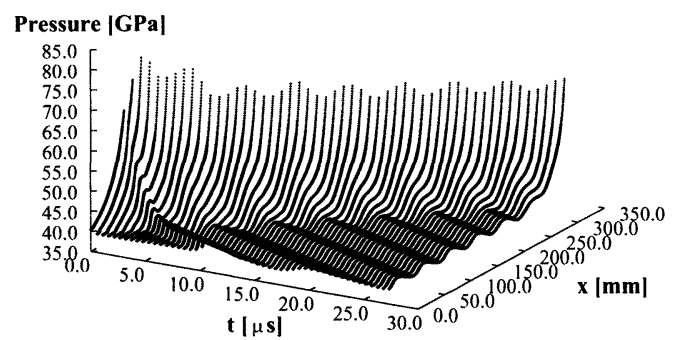

FIGURE 3. Pressure profiles $(\sigma=1.0$ and $\Pi=2.0)$.

The effect of the spatial frequency $\alpha$ was examined for several values of the amplitude $\sigma$. Rather than the spatial frequency, it is more convenient to refer to $\Pi$, the number of periods within the reaction zone. We have analyzed the results by computing the average, maximum, and minimum shock wave speed for the latter portion of the simulation after the initial transient has settled down. These values are depicted vs. $\sigma$ in Fig. 4 through 7 for $\Pi=2.00,1.00,0.50$, 0.25 . As expected, the average velocity converges to the uniform rate velocity as $\sigma$ goes to zero.

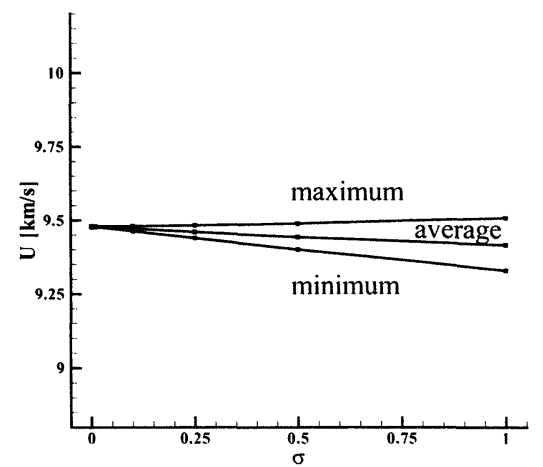

FIGURE 4. Front velocities for $\Pi=2.00$.

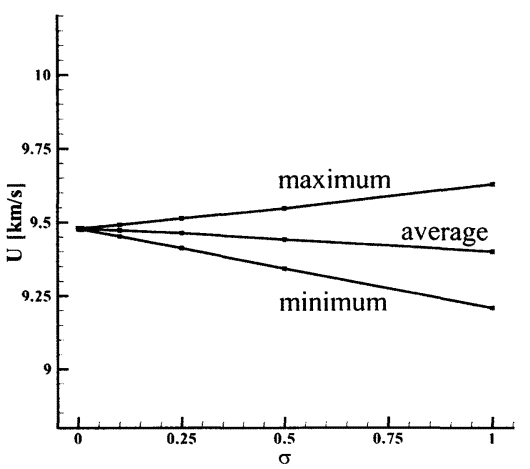

FIGURE 5. Front velocities for $\Pi=1.00$.

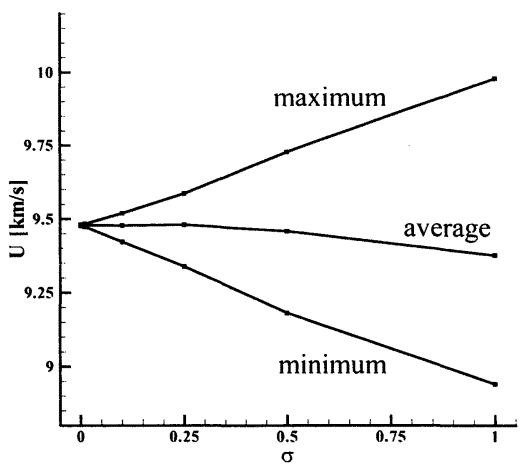

FIGURE 6. Front velocities for $\Pi=0.50$. 


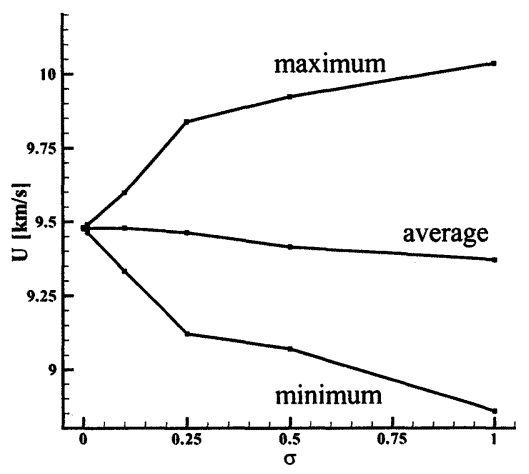

FIGURE 7. Front velocities for $\Pi=0.25$.

We also observe in Fig. 8 that the difference $\Delta U=\left(U^{\max }-U^{\min }\right)$ decreases with increasing $\Pi$. Unfortunately, for longer wavelengths $\Pi \leq 0.25$ the initial transient lasts for most of the computation and the results are not reliable.

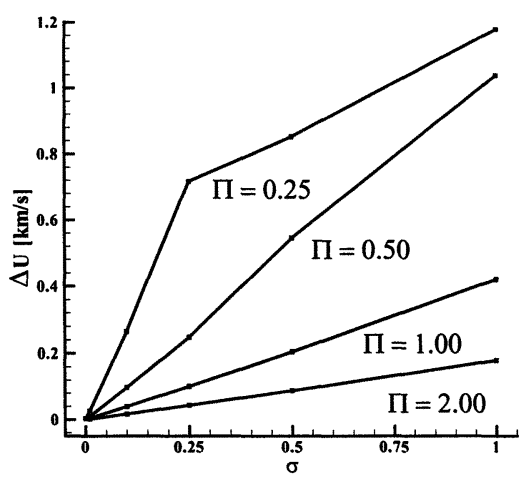

FIGURE 8. Front velocity amplitudes.

\section{CONCLUSIONS}

A simple model of heterogeneity in reaction rate has been investigated using a model equation of state that mimics high-explosive properties. A periodic disturbance in reaction rate does produce oscillations in the shock amplitude and acoustic waves are emitted in the downstream direction. The remarkable result is that even for large amplitude shock $(\sim 10 \%)$ oscillations, the average shock velocity remains within $2 \%$ of C.J velocity for the case we examined. Short wavelength disturbances resulted in much lower amplitude shock oscillations than long wavelength disturbances. This suggests that in simulating actual materials with wide range of scales, it is most important to model variations in rate on a scale of multiple reaction zone lengths rather than resolving fine structure within the reaction zone.

It is important to note that the velocity defects are very small and extreme care is needed to obtain reliable values. Although we have examined the issues of grid convergence and the influence of the rear boundary condition, we are not completely satisfied that these have been resolved. However, we believe that the trends are reliable. Our conclusion is that even in the presence of reaction rate heterogeneity, the CJ model is still a remarkably good theory for the estimation of average propagation speed. Apparently the amount of energy propagated away by the unsteady acoustic disturbances is extremely small for the cases that we have examined.

Work on this model continues and we are examining two-dimensional situations such as corner turning, initiation, stochastic placement of the hot-spots and feedback between hot-spot parameter and shock strength.

\section{ACKNOWLEDGMENTS}

This work was carried out at Caltech ASCI ASAP and funded by Contract B341492 under DOE Contract W-7405-ENG-48.

\section{REFERENCES}

1. Arienti, M., Morano, E., and Shepherd, J.E., Caltech Res. Rep. FM99-8 (1999)

2. Field, J.E., Acc. Chem. Res., 25, 489-496 (1992)

3. Lee, E.L., and Tarver, C.M., Phys. of Fl., 23, 2362-2372 (1980)

4. Johnson, J.N., Tang, P.K., and Forest, C.A., J. of Appl. Phys., 57, 4323-4334 (1985)

5. Glaister, P., J. of Comp. Phys., 74, 382-408 (1988)

6. Strang, G., SIAM J. on Num. Anal. 5, 506-517 (1968)

7. Miller, G.H., and Puckett, E.G., J. of Comp. Phys., 128, 134-164 (1996) 